\section{Minimal Invasive Approach should become the Standard for the Management of Zinner Syndrome}

Received: November 12, 2015, Accepted: November 16, 2015, Published: November 24,2015

The presence of a renal agenesis with an ipsilateral seminal vesicle cyst and an ejaculatory duct obstruction is a rare congenital anomaly initially reported by Zinner in 1914 [1]. This anomaly can be responsible of perineal pain, hemospermia, hematuria, post-ejaculatory pain, urinary tract infection, infertility and lower urinary tract symptoms (LUTS).

It is well established that in case of symptomatology, surgery is the treatment of choice. Herein, we present a case of a laparoscopic en bloc resection of the left kidney, ureter and seminal vesicle in a 17 years old boy presenting a variant of Zinner syndrome with the left ureter draining in the ipsilateral seminal vesicle. The diagnosis was made by an abdominopelvic MRI after persistent fever and LUTS (Figure 1).

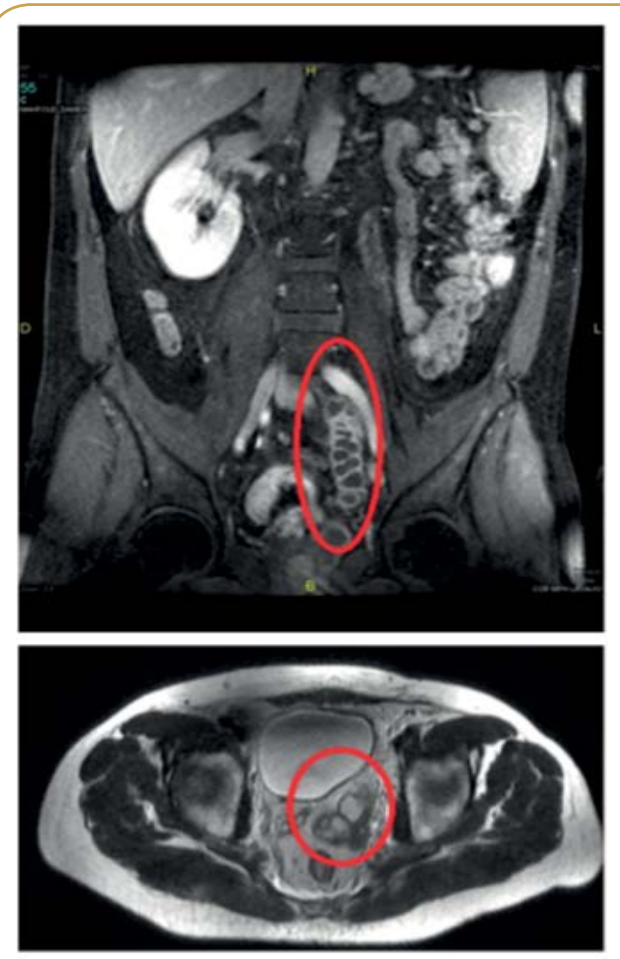

Figure 1 Coronal T1-weighted image (1a) after injection of Gadolinium showing an enhancement of the cyst wall with internal septations delineating hypointense fluid cavity and Axial T2-weighted (1b) images demonstrating the limits of the cyst with the bladder wall.

\section{Anthony Kallas Chemaly ${ }^{1}$, Fouad Aoun², Alexandire Peltier², Maroun Moukarzel ${ }^{3}$ and Henri Steyaert ${ }^{1}$}

\section{Department of pediatric urology, Queen Fabiola Children's University Hospital, Université libre de Bruxelles, Belgium \\ 2 Department of urology, Jules Bordet Institute, Université libre de Bruxelles, Belgium \\ 3 Department of urology, Hôtel-Dieu de France Hospital, Université Saint-Joseph, Liban}

\section{Corresponding author: \\ Alexandre Peltier \\ झ alexandre.peltier@bordet.be}

\begin{abstract}
Department of Urology, Jules Bordet Institute, Université libre de Bruxelles, Belgium.
\end{abstract}

Tel: 3225413111

\section{Discussion}

Various techniques have been proposed for the operative management of patients with Zinner syndrome.

Open resection for young fit patient and transurethral resection as well as transrectal aspiration for unfit patients have been proposed and different approaches such as transvesical, transperineal, transperitoneal, retroperitoneal and transcoccygeal approaches have been described [2].

However, these approaches are challenging and associated with a high risk of morbidity [3]. The difficulty stems mainly from the deep location of the seminal vesicle in the retrovesical region. Additionally, open access demands a large incision with extensive dissection of the bladder and exposes the patient to the risk of erectile dysfunction and rectal injury. Furthermore, some of these approaches are limited by the inability to treat upper urinary tract anomalies.

Recently, several authors had reported their experience with 
laparoscopic and robotic-assisted laparoscopic approach to treat patients with Zinner syndrome [4,5].

In our case, laparoscopic approach has been associated with minimal blood loss, quick convalescence, minimal postoperative pain and a good cosmetic result.

In our opinion, due to the complex anomalies encountered in this syndrome, laparoscopic transperitoneal approach should be the gold standard for treating these patients.
It allows direct access to the kidney, ureter and seminal vesicle by only incising the peritoneum. A fine and precise dissection and a good view would limit the blood loss, avoid damage to the bladder, rectum and neurovascular bundles.

We recommend always a multiplanar MRI before the surgery for evaluating the local extent of these seminal vesicle anomalies and to delineate the pathway of the ureter. 


\section{References}

1 Zinner A (1914) Ein Fall Von intravesikaler Samenblasenzyste. Wein Med Wochenschr 64: 605.

2 Okoye Bo, Jones DJ, Lancashire MJ, Brown EF, Ritchie AW (1995) Transvesical endoscopic drainage of a seminal vesicle cyst. Br J Urol 76: 810 .

3 Van den Ouden D, Blom JH, Bangma C, Spiegeleer AH (1998) Diagnosis and management of seminal vesicle cysts associated with ipsilateral renal agenesis: a pooled analysis of 52 cases. Eur Urol 33: 433-440

4 Cherullo EE, Meraney AM, Bernstein LH, Einstein DM, Thomas AJ, et al. (2002) Laparoscopic management of congenital seminal vesicle cysts associated with ipsilateral renal agenesis. J Urol 167: 1263-1267.

5 Kamlesh S, Bipinchandra P, Rizvi SJ, Pranjal M (2011) Laparoscopic excision of a congenital seminal vesicle cyst associated with ipsilateral renal agenesis. Urol Int 87: 238-240. 